\title{
Synthesis and Spectral Characterization of Novel 2-Pyrazoline and Bis-2-Pyrazoline Containing Quinoline Moiety
}

\author{
Allaoua Kedjadja ${ }^{1,2}$, Abdelmalek Bouraiou ${ }^{3}$, Rachid Merdes ${ }^{1}$ \\ ${ }^{1}$ Laboratoire de chimie Appliquée, Faculté des Mathématiques et de l'Informatique et des Sciences de la Matière, Université de \\ Guelma, Guelma, Algeria \\ ${ }^{2}$ Département de Science de la Matière, Faculté des Science, Université 20 Aout 1955 Skikda, Skikda, Algeria \\ ${ }^{3}$ Unité de Recherche de Chimie de l'Environnement et Moléculaire Structurale, Université des Frères Mentouri, Route de Ain El \\ Bey, Constantine, Algeria \\ Email: *kedjadjaalla@yahoo.fr
}

How to cite this paper: Kedjadja, A., Bouraiou, A. and Merdes, R. (2018) Synthesis and Spectral Characterization of Novel 2-Pyrazoline and Bis-2-Pyrazoline Containing Quinoline Moiety. International Journal of Organic Chemistry, 8, 105-114. https://doi.org/10.4236/ijoc.2018.81006

Received: December 3, 2017

Accepted: March 4, 2018

Published: March 7, 2018

Copyright (c) 2018 by authors and Scientific Research Publishing Inc. This work is licensed under the Creative Commons Attribution International License (CC BY 4.0).

http://creativecommons.org/licenses/by/4.0/

\begin{abstract}
Eight new compounds containing in their structures substituted quinoline and pyrazole entity were synthesized in good to excellent yield by cyclocondensation reaction of chalcones and hydrazine hydrate. This reaction was conducted in formic acid in presence of $\mathrm{BF}_{3} \cdot \mathrm{Et}_{2} \mathrm{O}$ or in acetic acid. These approaches were extended to bis-chalcones, which delivered functionalized bispyrazolines. The structures of the prepared compounds were confirmed by IR, ${ }^{1} \mathrm{HNMR},{ }^{13} \mathrm{CNMR}$ and mass spectral analysis.
\end{abstract}

Keywords

Pyrazoline, Chalcone, Quinoline, Cyclocondensation

\section{Introduction}

This quinoline nucleus is an important class of heterocyclic structure found in many synthetic and natural occurring products with a wide range of pharmacological activities [1], such as antiviral [2], antimalarial [3], anticancer [4], antibacterial [5], antifungal [6], antiobesity [7] and anti-inflammatory [8]. These properties are well illustrated by a large number of commercially available drugs containing this heterocyclic system. The $\alpha, \beta$-unsaturated ketones (chalcones) are found as naturally-occurring compounds and are considered to be the precursors of flavonoids and is of lavonoids. Chalcones are important targets medicinal chemistry due to their useful biological activities such as anti-inflammatory 
[9] [10] [11], antimitotic [12], anti-leishmanial [13], anti-invasive [14] [15], anti-tuberculosis [16], anti-fungal [17], anti-malarial [18] [19], anti-tumor and anti-oxidant properties [20], as well as their recognized synthetic utility in the preparation of pharmacologically-interesting heterocyclic systems.

In other hand, pyrazolines have also been primarily studied owing to their pharmacological activities, which include anti-tumor [21], anti-inflammatory [22], anti-parasitic [23], anti-depressive, anticonvulsant [24], antimicrobial [25], antitubercular [26] and nitric oxide synthase inhibitors, associated with diseases such as Alzheimer, Huntington, and inflammatory arthritis [27].

Numerous research programs have been devoted to the establishment of new synthetic methods and new heterocyclic combination because several biological active compounds contain a heterocyclic moiety as a fundamental subunit. In this context, we present in this paper an efficient and convenient protocol for the synthesis of novel 2-pyrazoline and bis-2-pyrazoline. These compounds were prepared by cyclocondensation of chalcones and bis-chalcones in presence of hydrazine hydrate. The new prepared compounds will contain both quinoline and pyrazoline moieties.

\section{Experimental}

\subsection{Material and Methods}

Melting points of prepared compounds were determined in open capillary tube M.P. apparatus expressed in ${ }^{\circ} \mathrm{C}$ and were uncorrected. Chemicals and solvents were of highest purity commercially available. All IR spectra were performed on Shimadzu FT-IR-8201 PC spectrophotometer. ${ }^{1} \mathrm{H}-\mathrm{NMR}$ and ${ }^{13} \mathrm{C}-\mathrm{NMR}$ spectra were recorded on Brüker Avance 250 and 500 spectrometers. Chemical shifts are given in ppm and J values in Hertz $(\mathrm{Hz})$. Mass spectra were recorded on a Shimadzu GCMS-QP2010. Low resolution mass spectra were recorded on Agilent 5975B inert Series GC/MS System. Column chromatography was performed on Merck silica gel (60, particle size $0.063-0.2 \mathrm{~mm}$ ). Thin-layer chromatography (TLC) was carried out on precoated Merck silica-gel aluminium sheets 60 F254.

\subsection{Synthesis}

\subsubsection{General Procedure for the Synthesis of Quinolines (1) and Chalcones (2)}

Substituted 3-Acetyl-2-methylquinoline derivatives $1 \mathrm{a}$ and $1 \mathrm{~b}$ have been prepared in accordance with established methods [28]. Spectroscopic results and physical properties are in agreement with literature reports [29] [30]. Chalcones $2 \mathbf{a}$ and $\mathbf{2 b}$ prepared from the method reported in literature [30] and their structures have been confirmed by spectroscopic methods.

\subsubsection{General Procedure for the Synthesis of Bis-Chalcones (3)}

A solution of terephthalaldehyde $(0.01 \mathrm{~mol})$ and substituted quinoline1 (0.02 $\mathrm{mol}$ ) in $20 \mathrm{ml}$ of alcohol, were added slowly $0.02 \mathrm{~mol}$ sodium hydroxide solution. The mixture was stirred magnetically for $6 \mathrm{hr}$. Then the mixture was 
poured slowly into ice $(200 \mathrm{~g})$ and the solution was adjusted to $\mathrm{pH} \sim 2$ by $\mathrm{HCl}(1$ $\mathrm{N})$ with constant stirring then kept in refrigerator for 12 hours. The precipitate obtained filtered, washed and recrystallized from chloroform/ethyl acetate mixture (ratio $1 / 1)$.

(2E,2'E)-1,1'-Bis(2-methyl-4-phenylquinolin-3-yl)-3,3'-(1,4-phenylene)di prop-2-en-1-one (3a): White solid, Yield $=84 \%, \mathrm{~m} . \mathrm{p}>280^{\circ} \mathrm{C}$, IR $\left(\mathrm{KBr}, \mathrm{cm}^{-1}\right)$ : $1604.7(\mathrm{C}=\mathrm{O}) .{ }^{1} \mathrm{H}-\mathrm{NMR}\left(500 \mathrm{MHz}, \mathrm{CDCl}_{3}, \delta \mathrm{ppm}, \mu \mathrm{Hz}\right): 8.10(\mathrm{~d}, J=8.9,2 \mathrm{H}$, $\mathrm{H}-8$ ), 7.90 - 7.20 (m, 20H, H-Ar), 7.08 (d, $J=16.2,2 \mathrm{H}, \mathrm{H} \beta), 6.68$ (d, $J=16.2,2 \mathrm{H}$, $\mathrm{H} \alpha), 2.70\left(\mathrm{~s}, 6 \mathrm{H}, 2 \mathrm{CH}_{3}\right) ;{ }^{13} \mathrm{C}-\mathrm{NMR}\left(125.7 \mathrm{MHz}, \mathrm{CDCl}_{3}, \delta \mathrm{ppm}\right): 196.79$ (2CO), $155.22,146.17,144.84,144.64,136.31,134.46,133.12,132.57,131.16,130.58$, $129.92,128.98,128.79,128.64,128.56,126.01,125.04(\mathrm{C}, \mathrm{CH}), 21.05\left(2 \mathrm{CH}_{3}\right)$.

(2E,2'E)-1,1'-Bis(6-chloro-2-methyl-4-phenylquinolin-3-yl)-3,3'-(1,4-phe nylene)diprop-2-en-1-one [31] (3b): Yellow needles, Yield $=75 \%, \mathrm{~m} . \mathrm{p}>$ $280^{\circ} \mathrm{C}$, IR $\left(\mathrm{KBr}, \mathrm{cm}^{-1}\right): 1604.7(\mathrm{C}=\mathrm{O}) .{ }^{1} \mathrm{H}-\mathrm{NMR}\left(500 \mathrm{MHz}, \mathrm{CDCl}_{3}, \delta \mathrm{ppm}, / \mathrm{Hz}\right)$ : $8.10(\mathrm{~d}, J=8.9,2 \mathrm{H}, \mathrm{H}-8), 7.90-7.20(\mathrm{~m}, 18 \mathrm{H}, \mathrm{H}-\mathrm{Ar}), 7.10$ (d, $J=16.2,2 \mathrm{H}, \mathrm{H} \beta)$, $6.68(\mathrm{~d}, J=16.2,2 \mathrm{H}, \mathrm{H} \alpha), 2.70\left(\mathrm{~s}, 6 \mathrm{H}, 2 \mathrm{CH}_{3}\right),{ }^{13} \mathrm{C}-\mathrm{NMR}\left(125.7 \mathrm{MHz}, \mathrm{CDCl}_{3}, \delta\right.$ ppm): 198.04 (2CO), 156.20, 147.10, 145.75, 145.56, 137.17, 135.31, 133.97, $133.41,131.99,131.40,130.74,129.80,129.60,129.45,129.37,126.81,125.83$ (C, $\mathrm{CH}), 21.18\left(\mathrm{CH}_{3}\right)$.

\subsubsection{General Procedure for the Synthesis of Pyrazolines (4)}

A mixture of chalcones $2(1.71 \mathrm{mmol})$, boron trifluoride diethyl etherate (3 drops) and hydrazine hydrate $(1.80 \mathrm{mmol})$ in DMF $(5 \mathrm{~mL})$, was heated to reflux for $6 \mathrm{~h}$. Formic acid $(10 \mathrm{~mL})$ was added to the reaction mixture and the refluxing was further continued for $2 \mathrm{~h}$. After cooling, the adding of crushed ice precipitated a solid which was filtered and washed thoroughly with water and dried at ambient temperature. The residue was subjected to column chromatography (silica gel, eluent: $\mathrm{CHCl}_{3}$ ) to afford pure products 4 .

3-(2-Methyl-4-phenylquinolin-3-yl)-5-phenyl-4,5-dihydro-1H-pyrazole1-carbaldehyde (4a): Yellow powder, Yield $=86 \%, \mathrm{~m} . \mathrm{p} .=204^{\circ} \mathrm{C}, \mathrm{IR}(\mathrm{KBr}$, $\left.\mathrm{cm}^{-1}\right)$ : $1684.1(\mathrm{C}=\mathrm{O}), 1620.8(\mathrm{C}=\mathrm{N}), 1582.4(\mathrm{C}=\mathrm{C}) .{ }^{1} \mathrm{H}-\mathrm{NMR}\left(500 \mathrm{MHz}, \mathrm{CDCl}_{3}\right.$, $\delta \mathrm{ppm}, / \mathrm{Hz}): 8.58(\mathrm{~s}, 1 \mathrm{H}, \mathrm{CHO}), 7.72(\mathrm{~d}, J=8.3 \mathrm{~Hz}, 1 \mathrm{H}, \mathrm{H}-8), 7.55(\mathrm{~d}, J=8.3 \mathrm{~Hz}$, 1H, H-5), $7.40-7.05$ (m, 10H, H-Ar), $6.85-6.75$ (m, 2H, H-Ar), 5.29 (dd, $J=$ $11.9 \mathrm{~Hz}, J=5.1 \mathrm{~Hz}, 1 \mathrm{H}, \mathrm{H}-5$ pyrazoline), $3.85(\mathrm{dd}, J=18.6 \mathrm{~Hz}, J=12.0 \mathrm{~Hz}, 1 \mathrm{H}$, $\mathrm{H}-4$ pyrazoline), 3.16 (dd, $J=18.5 \mathrm{~Hz}, J=5.1 \mathrm{~Hz}, 1 \mathrm{H}, \mathrm{H}-4$ ' pyrazoline), 2.22 (s, $\left.3 \mathrm{H}, \mathrm{CH}_{3}\right) .{ }^{13} \mathrm{C}-\mathrm{NMR}\left(125.7 \mathrm{MHz}, \mathrm{CDCl}_{3}, \delta \mathrm{ppm}\right): 162.50(\mathrm{CHO}), 155.25(\mathrm{C}=\mathrm{N}$, C2 quinoline), 146.20 (C=N, C3 pyrazoline), 144.86, 144.67, 136.33, 134.48, $133.15,132.60,131.19,130.60,129.94,129.00,128.81,128.66,128.58,126.03$, 125.06, (C, $\mathrm{CH}$ phenyl and quinoline, 20C), $60.40(\mathrm{CH}, \mathrm{C} 5$ pyrazoline), 45.55 $\left(\mathrm{CH}_{2}, \mathrm{C} 4\right.$ pyrazoline), $23.96\left(\mathrm{CH}_{3}\right)$. MS (EI): $\mathrm{m} / \mathrm{z} 391\left(\mathrm{M}^{+}, 100\right), 363(64), 339$ (17), 327 (10), 312 (18), 290 (78), 270 (48), 117 (25), 103 (15), 91 (26), 77 (43), 65 (28), 51 (22).

3-(6-chloro-2-Methyl-4-phenylquinolin-3-yl)-5-phenyl-4,5-dihydro-1Hpyrazole-1-carbaldehyde (4b): Yellow powder, Yield $=92 \%, \mathrm{~m} . \mathrm{p} .=212^{\circ} \mathrm{C}, \mathrm{IR}$ 
$\left(\mathrm{KBr}, \mathrm{cm}^{-1}\right): 1684.1(\mathrm{C}=\mathrm{O}), 1620.8(\mathrm{C}=\mathrm{N}), 1582.4(\mathrm{C}=\mathrm{C}) .{ }^{1} \mathrm{H}-\mathrm{NMR}(500 \mathrm{MHz}$, $\left.\mathrm{CDCl}_{3}, \delta \mathrm{ppm}, / \mathrm{Hz}\right): 8.60$ (s, 1H, CHO), $7.72(\mathrm{~d}, J=8.3 \mathrm{~Hz}, 1 \mathrm{H}, \mathrm{H}-8), 7.50-7.05$ (m, 10H, H-Ar), $6.90-6.70$ (m, 2H, H-Ar), 5.35 (dd, J=11.9 Hz, J=5.1 Hz, $1 \mathrm{H}$, H-5 pyrazoline), 3.84 (dd, $J=18.6 \mathrm{~Hz}, J=12.0 \mathrm{~Hz}, 1 \mathrm{H}, \mathrm{H}-4$ pyrazoline), 3.16 (dd, $J=18.5 \mathrm{~Hz}, J=5.1 \mathrm{~Hz}, 1 \mathrm{H}, \mathrm{H}-4$ ' pyrazoline), $2.84\left(\mathrm{~s}, 3 \mathrm{H}, \mathrm{CH}_{3}\right) .{ }^{13} \mathrm{C}-\mathrm{NMR}$ (125.7 MHz, $\left.\mathrm{CDCl}_{3}, \delta \mathrm{ppm}\right)$ : $161.82(\mathrm{CHO}), 156.20$ (C=N, C2quinoline), 147.10 (C=N, C3pyrazoline), 145.75, 145.56, 137.7, 135.31, 133.97, 133.41, 131.99, 131.40, 130.74, 129.80, 129.60, 129.45, 129.37, 126.81, 125.83, (C, CH phenyl and quinoline, 20C), 63.97 ( $\mathrm{CH}$, C5 pyrazoline), $45.83\left(\mathrm{CH}_{2}, \mathrm{C} 4\right.$ pyrazoline), 24.11 $\left(\mathrm{CH}_{3}\right)$.

\subsubsection{General Procedure for the Synthesis of Pyrazolines (5)}

A mixture of chalcones $2(1.71 \mathrm{mmol})$, hydrazine hydrate $(1.8 \mathrm{mmol})$ and acetic acid $(10.0 \mathrm{~mL})$ was heated under reflux for $6 \mathrm{~h}$ until complete consumption of the chalcone (TLC control). After cooling, the resulting solution was neutralized with concentrate ammonium hydroxide. Then, the adding of crushed ice to the solution precipitated a solid which was filtered and washed with water. Pure compounds 5 was obtained by crystallization from chloroform/ethyl acetate mixture (1:2).

1-[3-(2-methyl-4-phenylquinolin-3-yl)-5-(phenyl)-4,5-dihydro-1H-pyraz ole-1-yl] ethanone (5a):White powder, Yield $=87 \%, \mathrm{~m} . \mathrm{p} .=212^{\circ} \mathrm{C}, \mathrm{IR}(\mathrm{KBr}$, $\left.\mathrm{cm}^{-1}\right)$ : $1674.1(\mathrm{C}=\mathrm{O}), 1620.1(\mathrm{C}=\mathrm{N}), 1581.5(\mathrm{C}=\mathrm{C}) .{ }^{1} \mathrm{H}-\mathrm{NMR}\left(250 \mathrm{MHz}, \mathrm{CDCl}_{3}\right.$, $\delta \mathrm{ppm}, J \mathrm{~Hz}): 8.13(\mathrm{~d}, J=8.3 \mathrm{~Hz}, 1 \mathrm{H}, \mathrm{H}-8), 7.75(\mathrm{td}, J=8.3 \mathrm{~Hz}, J=1.8 \mathrm{~Hz}, 1 \mathrm{H}$, H-7), $7.53-7.25$ (m, 10H, H-Ar), $6.90-6.84$ (m, 2H, H-Ar), 5.35 (dd, $J=11.9$ $\mathrm{Hz}, J=5.1 \mathrm{~Hz}, 1 \mathrm{H}, \mathrm{H}-5$ pyrazoline), 3.44 (dd, $J=18.6 \mathrm{~Hz}, J=12.0 \mathrm{~Hz}, 1 \mathrm{H}, \mathrm{H}-4$ pyrazoline), $2.82\left(\mathrm{~s}, 3 \mathrm{H}, \mathrm{CH}_{3}\right), 2.53(\mathrm{dd}, J=18.5 \mathrm{~Hz}, J=5.1 \mathrm{~Hz}, 1 \mathrm{H}, \mathrm{H}-4$ ' pyrazoline), $2.18\left(\mathrm{~s}, 3 \mathrm{H}, \mathrm{CH}_{3}\right) .{ }^{13} \mathrm{C}-\mathrm{NMR}\left(62.9 \mathrm{MHz}, \mathrm{CDCl}_{3}, \delta \mathrm{ppm}\right): 172.50$ (CO), $156.57(\mathrm{C}=\mathrm{N}, \mathrm{C} 2$ quinoline $), 153.82(\mathrm{C}=\mathrm{N}, \mathrm{C} 3$ pyrazoline $), 148.28,147.55$, $141.62,135.77,130.40,130.09,129.64,128.87,128.70,128.57,127.54,126.58$, $126.42,125.80,125.62,124.74(\mathrm{C}, \mathrm{CH}$ phenyl and quinoline, 20C), $59.69(\mathrm{CH}, \mathrm{C5}$ pyrazoline), $46.51\left(\mathrm{CH}_{2}, \mathrm{C} 4\right.$ pyrazoline), $27.83\left(\mathrm{CH}_{3}\right), 24.81\left(\mathrm{CH}_{3}\right)$. MS (EI): $\mathrm{m} / \mathrm{z}$ 405 ( $\left.\mathrm{M}^{+}, 100\right), 390$ (29), 362 (32), 322 (10), 286 (79), 221 (19), 213 (46), 198 (21), 170 (20), 142 (10), 110 (19), 70 (8), 22 (14).

1-[3-(6-chloro-2-methyl-4-phenylquinolin-3-yl)-5-(phenyl)-4,5-dihydro$1 \mathrm{H}$-pyrazole-1-yl]ethanone (5b): Yellow powder, Yield $=82 \%, \mathrm{~m} . \mathrm{p} .=223^{\circ} \mathrm{C}$, IR $\left(\mathrm{KBr}, \mathrm{cm}^{-1}\right)$ : $1682.4(\mathrm{C}=\mathrm{O}), 1623.8(\mathrm{C}=\mathrm{N}), 1585.2(\mathrm{C}=\mathrm{C}) .{ }^{1} \mathrm{H}-\mathrm{NMR}(250 \mathrm{MHz}$, $\left.\mathrm{CDCl}_{3}, \delta \mathrm{ppm}, J \mathrm{~Hz}\right): 8.18(\mathrm{~d}, J=8.3 \mathrm{~Hz}, 1 \mathrm{H}, \mathrm{H}-8), 7.79(\mathrm{dd}, J=8.3 \mathrm{~Hz}, J=1.8$ $\mathrm{Hz}, 1 \mathrm{H}, \mathrm{H}-7$ ), 7.50 - 7.20 (m, 9H, H-Ar), $6.90-6.80$ (m, 2H, H-Ar), 5.32 (dd, $J=$ $11.9 \mathrm{~Hz}, J=5.1 \mathrm{~Hz}, 1 \mathrm{H}, \mathrm{H}-5$ pyrazoline), $3.42(\mathrm{dd}, J=18.6 \mathrm{~Hz}, J=12.0 \mathrm{~Hz}, 1 \mathrm{H}$, H-4 pyrazoline), $2.80\left(\mathrm{~s}, 3 \mathrm{H}, \mathrm{CH}_{3}\right), 2.51\left(\mathrm{dd}, J=18.5 \mathrm{~Hz}, J=5.1 \mathrm{~Hz}, 1 \mathrm{H}, \mathrm{H}-4^{\prime}\right.$ pyrazoline), $2.75\left(\mathrm{~s}, 3 \mathrm{H}, \mathrm{CH}_{3}\right) .{ }^{13} \mathrm{C}-\mathrm{NMR}\left(62.9 \mathrm{MHz}, \mathrm{CDCl}_{3}, \delta \mathrm{ppm}\right): 171.08$ (CO), 155.02 ( $\mathrm{C}=\mathrm{N}, \mathrm{C} 2$ quinoline), $151.65(\mathrm{C}=\mathrm{N}, \mathrm{C} 3$ pyrazoline), 147.78, 146.91, $145.61,135.27,134.14,132.70,131.03,130.30,130.10,129.03,128.92,128.70$, $128.48,126.65,126.45,125.40(\mathrm{C}, \mathrm{CH}$ phenyl and quinoline, 20C), $59.82(\mathrm{CH}, \mathrm{C} 5$ 
pyrazoline), $46.23\left(\mathrm{CH}_{2}, \mathrm{C} 4\right.$ pyrazoline $), 28.51\left(\mathrm{CH}_{3}\right), 23.99\left(\mathrm{CH}_{3}\right)$.

\subsubsection{General Procedure for the Synthesis of Bis-Pyrazolines (6)}

A mixture of bis-chalcones $3(0.96 \mathrm{mmol})$, boron trifluoride diethyl etherate (3 drops) and hydrazine hydrate $(2.0 \mathrm{mmol})$ in DMF $(8 \mathrm{~mL})$ was refluxed for $6 \mathrm{~h}$. Formic acid $(12 \mathrm{~mL})$ was added to the reaction mixture and the refluxing was further continued for $2 \mathrm{~h}$. After cooling, the adding of crushed ice precipitated a solid which was filtered and washed thoroughly with water and dried at ambient temperature. The residue was subjected to column chromatography (silica gel, eluent: $\mathrm{CHCl}_{3}$ ) to afford pure products 6 .

1,1'-\{1,4-phenylenebis [3-(2-methyl-4-phenylquinolin-3-yl)-4,5-dihydro$1 H$-pyrazole-5,1-diyl] $\}$ dicarbaldehyde (6a):Yellow solid, Yield $=86 \%$, m.p. $>$ $280^{\circ} \mathrm{C}$, IR $\left(\mathrm{KBr}, \mathrm{cm}^{-1}\right): 1689.5(\mathrm{C}=\mathrm{O}), 1620.1(\mathrm{C}=\mathrm{N}), 1581.5(\mathrm{C}=\mathrm{C}) .{ }^{1} \mathrm{H}-\mathrm{NMR}$ $\left(250 \mathrm{MHz}, \mathrm{CDCl}_{3}, \delta \mathrm{ppm}, / \mathrm{Hz}\right): 8.90$ (s, 2H, 2CHO), 8.66 (d, $J=8.4 \mathrm{~Hz}, 2 \mathrm{H}, \mathrm{H}-8$ quinoline), 8.28 (dd, $J=8.4 \mathrm{~Hz}, J=1.7 \mathrm{~Hz}, 2 \mathrm{H}, \mathrm{H}-7$ quinoline), $8.07-7.91(\mathrm{~m}$, 10H, H-Ar), 7.87 - 7.79 (m, 4H, H-Ar), 7.24 (s, 4H, phenylene), 5.86 (dd, $J=12.0$ $\mathrm{Hz}, J=5.0 \mathrm{~Hz}, 2 \mathrm{H}, \mathrm{H}-5$ pyrazoline), $3.64(\mathrm{dd}, J=18.4 \mathrm{~Hz}, J=12.0 \mathrm{~Hz}, 2 \mathrm{H}, \mathrm{H}-4$ pyrazoline), $3.36\left(\mathrm{~s}, 6 \mathrm{H}, 2 \mathrm{CH}_{3}\right), 2.98(\mathrm{dd}, J=18.4 \mathrm{~Hz}, J=5.0 \mathrm{~Hz}, 2 \mathrm{H}, \mathrm{H}-4$ ' pyrazoline). ${ }^{13} \mathrm{C}$-NMR (62.9 MHz, $\left.\mathrm{CDCl}_{3}, \delta \mathrm{ppm}\right): 159.44$ (2CHO), $156.55(2 \mathrm{C}=\mathrm{N} \mathrm{C} 2$ quinoline), 154.07 ( $2 \mathrm{C}=\mathrm{N}, \mathrm{C} 3$ pyrazoline), 148.43, 147.52, 140.72, 135.67, 130.48, $130.02,129.60,128.87,128.76,128.65,126.64,126.48,126.04,125.80,124.68$ (C, $\mathrm{CH}$ phenyl and quinoline, 34C), $59.28(2 \mathrm{CH}$, $\mathrm{C} 5$ pyrazoline $), 46.30\left(2 \mathrm{CH}_{2}, \mathrm{C} 4\right.$ pyrazoline), $24.87\left(2 \mathrm{CH}_{3}\right)$. MS (EI): m/z $704\left(\mathrm{M}^{+}, 100\right), 652(19), 648$ (78), 598 (31), 556 (13), 494 (62), 445 (17), 398 (19), 358 (21), 306 (15), 256 (11), 200 (10), $173(8), 120(9), 38(10)$

1,1'-\{1,4-phenylenebis [3-(6-chloro-2-methyl-4-phenylquinolin-3-yl)-4,5dihydro- $1 H$-pyrazole-5,1-diyl] $\}$ dicarbaldehyde (6b): White powder, Yield $=$ 78\%, m.p. > 280 ${ }^{\circ} \mathrm{C}, \mathrm{IR}\left(\mathrm{KBr}, \mathrm{cm}^{-1}\right): 1689.5(\mathrm{C}=\mathrm{O}), 1620.1(\mathrm{C}=\mathrm{N}), 1581.5(\mathrm{C}=\mathrm{C})$. ${ }^{1} \mathrm{H}-\mathrm{NMR}\left(250 \mathrm{MHz}, \mathrm{CDCl}_{3}, \delta \mathrm{ppm}, / \mathrm{Hz}\right): 8.98(\mathrm{~s}, 2 \mathrm{H}, 2 \mathrm{CHO}), 8.50$ (d, $J=8.4 \mathrm{~Hz}$, $2 \mathrm{H}, \mathrm{H}-8$ quinoline), 8.28 (dd, $J=8.4 \mathrm{~Hz}, J=1.7 \mathrm{~Hz}, 2 \mathrm{H}, \mathrm{H}-7$ quinoline), $8.10-7.70$ (m, 14H, H-Ar), 7.24 (s, $4 \mathrm{H}$, phenylene), $5.85(\mathrm{dd}, J=12.0 \mathrm{~Hz}, J=5.0 \mathrm{~Hz}, 2 \mathrm{H}$, H-5 pyrazoline), 3.65 (dd, $J=18.4 \mathrm{~Hz}, J=12.0 \mathrm{~Hz}, 2 \mathrm{H}, \mathrm{H}-4$ pyrazoline), 3.34 (s, $\left.6 \mathrm{H}, 2 \mathrm{CH}_{3}\right), 2.96\left(\mathrm{dd}, J=18.4 \mathrm{~Hz}, J=5.0 \mathrm{~Hz}, 2 \mathrm{H}, \mathrm{H}-4\right.$ ' pyrazoline). ${ }^{13} \mathrm{C}-\mathrm{NMR}$ (62.9 $\left.\mathrm{MHz}, \mathrm{CDCl}_{3}, \delta \mathrm{ppm}\right): 160.14(2 \mathrm{CHO}), 156.20$ (2C=N, C2 quinoline), $155.61(2 \mathrm{C}=\mathrm{N}, \mathrm{C} 3$ pyrazoline $), 149.82,147.65,142.21,138.93,135.19,132.36$, $131.07,129.99,129.56,128.91,127.67,127.50,126.79,125.94,121.73(\mathrm{C}, \mathrm{CH}$ phenyl and quinoline, $34 \mathrm{C}), 59.67$ (2CH, C5 pyrazoline), $47.23\left(2 \mathrm{CH}_{2}, \mathrm{C} 4\right.$ pyrazoline), $21.73\left(2 \mathrm{CH}_{3}\right)$. HRMS (ESI) $\mathrm{m} / \mathrm{zcalcd}$ for $\mathrm{C}_{46} \mathrm{H}_{34} \mathrm{Cl}_{2} \mathrm{~N}_{6} \mathrm{O}_{2}[\mathrm{M}+\mathrm{Na}]^{+}=$ 795.2054, found 795.2018.

\subsubsection{General Procedure for the Synthesis of Bis-Pyrazolines (7)}

A mixture of bis-chalcones $3(0.96 \mathrm{mmol})$, hydrazine hydrate $(2 \mathrm{mmol})$ and acetic acid $(10.0 \mathrm{~mL})$ was heated under reflux for $6 \mathrm{~h}$. After cooling, the resulting solution was neutralized with concentrate ammonium hydroxide. The adding of 
crushed ice to the solution precipitated a solid which was filtered and washed with water. Pure compounds 7 was obtained by crystallization from chloroform/ ethyl acetate mixture $(1: 1)$.

1,1'-\{1,4-phenylenebis [3-(2-methyl-4-phenylquinolin-3-yl)-4,5-dihydro$1 H$-pyrazole-5,1-diyl]\}diethanone (7a): Brown solid, Yield $=73 \%$, m.p. $>$ $280^{\circ} \mathrm{C}$, IR $\left(\mathrm{KBr}, \mathrm{cm}^{-1}\right): 1666.4(\mathrm{C}=\mathrm{O}), 1620.1(\mathrm{C}=\mathrm{N}), 1581.5(\mathrm{C}=\mathrm{C}),{ }^{1} \mathrm{H}-\mathrm{NMR}$ (500 MHz, DMSO- $d_{\sigma} \delta \mathrm{ppm}, / \mathrm{Hz}$ ): 8.20 (d, $J=8.4 \mathrm{~Hz}, 2 \mathrm{H}, \mathrm{H}-8$ quinoline), 8.00 (dd, $J=8.4 \mathrm{~Hz}, J=1.7 \mathrm{~Hz}, 2 \mathrm{H}, \mathrm{H}-7$ quinoline), $7.80-7.30$ (m, 14H, H-Ar), 7.12 (s, $4 \mathrm{H}$, phenylene), $5.70(\mathrm{dd}, J=12.0 \mathrm{~Hz}, J=5.0 \mathrm{~Hz}, 2 \mathrm{H}, \mathrm{H}-5$ pyrazoline), 3.64 (dd, $J=18.4 \mathrm{~Hz}, J=12.0 \mathrm{~Hz}, 2 \mathrm{H}, \mathrm{H}-4$ pyrazoline), $3.30\left(\mathrm{~s}, 6 \mathrm{H}, 2 \mathrm{CH}_{3}\right.$ ), 2.88 (dd, $J$ $=18.4 \mathrm{~Hz}, J=5.0 \mathrm{~Hz}, 2 \mathrm{H}, \mathrm{H}-4$ ' pyrazoline $), 2.50\left(\mathrm{~s}, 6 \mathrm{H}, 2 \mathrm{CH}_{3}\right) .{ }^{13} \mathrm{C}-\mathrm{NMR}(125.7$ $\left.\mathrm{MHz}, \mathrm{DMSO}-d_{\sigma} \delta \mathrm{ppm}\right): 169.40$ (2CO), 159.40 (2C=N, C2 quinoline), 158.80 $(2 \mathrm{C}=\mathrm{N}, \mathrm{C} 3$ pyrazoline $), 144.30,142.80,140.70,137.10,132.50,129.70,128.80$, $128.60,126.40,126.00,125.80,124.60,123.40,118.80$ (C, $\mathrm{CH}$ phenyl and quinoline, $34 \mathrm{C}), 60.10\left(2 \mathrm{CH}\right.$, C5 pyrazoline), $45.70\left(2 \mathrm{CH}_{2}, \mathrm{C} 4\right.$ pyrazoline $), 27.30$ $\left(2 \mathrm{CH}_{3}\right), 24.60\left(2 \mathrm{CH}_{3}\right) . \mathrm{MS}(\mathrm{EI}): \mathrm{m} / \mathrm{z} 732\left(\mathrm{M}^{+}, 100\right), 702$ (77), 646 (32), 591 (23), 568 (72), 492 (21), 447 (20), 394 (19), 368 (12), 286 (14), 223 (12), 124 (14), 68 (19).

1,1'-\{1,4-phenylenebis [3-(6-chloro-2-methyl-4-phenylquinolin-3-yl)-4,5dihydro- $1 H$-pyrazole-5,1-diyl] $\}$ diethanone (7b): Yellow solid, Yield $=81 \%$, m.p. $>280^{\circ} \mathrm{C}$, IR $\left(\mathrm{KBr}, \mathrm{cm}^{-1}\right): 1639.4(\mathrm{C}=\mathrm{O}), 1620.1(\mathrm{C}=\mathrm{N}), 1566.1(\mathrm{C}=\mathrm{C})$. ${ }^{1} \mathrm{H}-\mathrm{NMR}\left(500 \mathrm{MHz}, \mathrm{DMSO}-d_{\circlearrowleft} \delta \mathrm{ppm}, / \mathrm{Hz}\right): 8.20(\mathrm{~d}, J=8.4 \mathrm{~Hz}, 2 \mathrm{H}, \mathrm{H}-8$ quinoline), 8.08 (dd, $J=8.4 \mathrm{~Hz}, J=1.7 \mathrm{~Hz}, 2 \mathrm{H}, \mathrm{H}-7$ quinoline), $7.80-7.30(\mathrm{~m}, 10 \mathrm{H}$, H-Ar), 7.12 (s, 4H, phenylene), 5.76 (dd, $J=12.0 \mathrm{~Hz}, J=5.0 \mathrm{~Hz}, 2 \mathrm{H}, \mathrm{H}-5$ pyrazoline), $3.64(\mathrm{dd}, J=18.4 \mathrm{~Hz}, J=12.0 \mathrm{~Hz}, 2 \mathrm{H}, \mathrm{H}-4$ pyrazoline), $3.36(\mathrm{~s}, 6 \mathrm{H}$, $\left.2 \mathrm{CH}_{3}\right), 2.88\left(\mathrm{dd}, J=18.4 \mathrm{~Hz}, J=5.0 \mathrm{~Hz}, 2 \mathrm{H}, \mathrm{H}-4\right.$ ' pyrazoline). ${ }^{13} \mathrm{C}$-NMR $(125.7$ $\left.\mathrm{MHz}, \mathrm{DMSO}-d_{\sigma} \delta \mathrm{ppm}\right): 169.78$ (2CO), 157.36 (2C, C2 quinoline), 155.3 (2C, C3 pyrazoline), 148.99, 146.83, 141.42, 138.16, 134.44, 131.62, 130.34, 129.57, $128.84,128.19,126.99,126.79,126.09,125.24$ (C, $\mathrm{CH}$ phenyl and quinoline, $34 \mathrm{C}), 60.96(2 \mathrm{CH}, \mathrm{C} 5$ pyrazoline $), 46.13\left(2 \mathrm{CH}_{2}, \mathrm{C} 4\right.$ pyrazoline $), 26.47\left(2 \mathrm{CH}_{3}\right)$, $21.61\left(2 \mathrm{CH}_{3}\right)$. HRMS (ESI) $\mathrm{m} / \mathrm{zcalcd}$ for $\mathrm{C}_{48} \mathrm{H}_{38} \mathrm{Cl}_{2} \mathrm{~N}_{6} \mathrm{O}_{2}[\mathrm{M}+\mathrm{Na}]^{+}=823.2331$, found 823.2348 .

\section{Result and Discussion}

Attempts have been made for the synthesis of $N$-acetyl substituted pyrazoline derivatives by reaction of hydrazine with chalcones possessing quinoline group. The routes to the target compounds 2, 3, 4, 5, 6 and 7 are shown in Schemes 1-3. 3-phenyl-1-(substitutedquinolin-3-yl)-2-propen-1-one (2a-b) and (2E, 2'E)-1,1'-bis(substitutedquinolin-3-yl)-3,3'-(1,4-phenylene)diprop-2-en-1-one (3a-b) were obtained via Claisen-Schmidt condensation from benzaldehyde and terephthalaldehyde using sodium hydroxide $(\mathrm{NaOH})$ as catalyst in ethanol. The result is excellent in terms of yield and product purity. Pyrazolines (4a-b and 5a-b) and bis-pyrazolines (6a-b and 7a-b) were obtained in a one-pot multicomponent reaction of chalcones 2 and 3 in presence of hydrazine hydrate in 


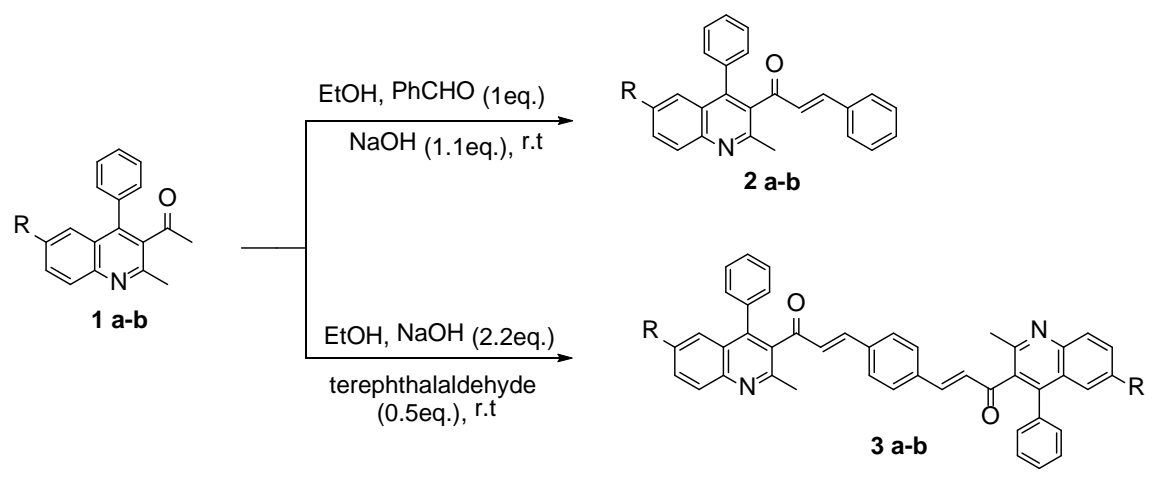

Scheme 1. Synthesis of chalcones 2 and bis-chalcones 3.

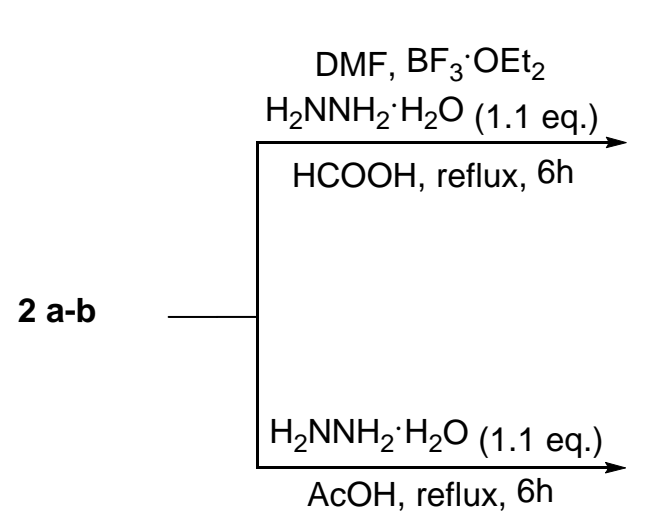<smiles>[R]c1ccc2nc(C)c(C3=NN(C=O)C(c4ccccc4)C3)c(-c3ccccc3)c2c1</smiles><smiles>[R5]CCCCC1CC(c2c(C)nc3ccc([R])cc3c2-c2ccccc2)=NN1C(C)=O</smiles>

Scheme 2. Synthesis of new $N$-formylpyrazolines 4 and $N$-acetyl pyrazolines 5 .

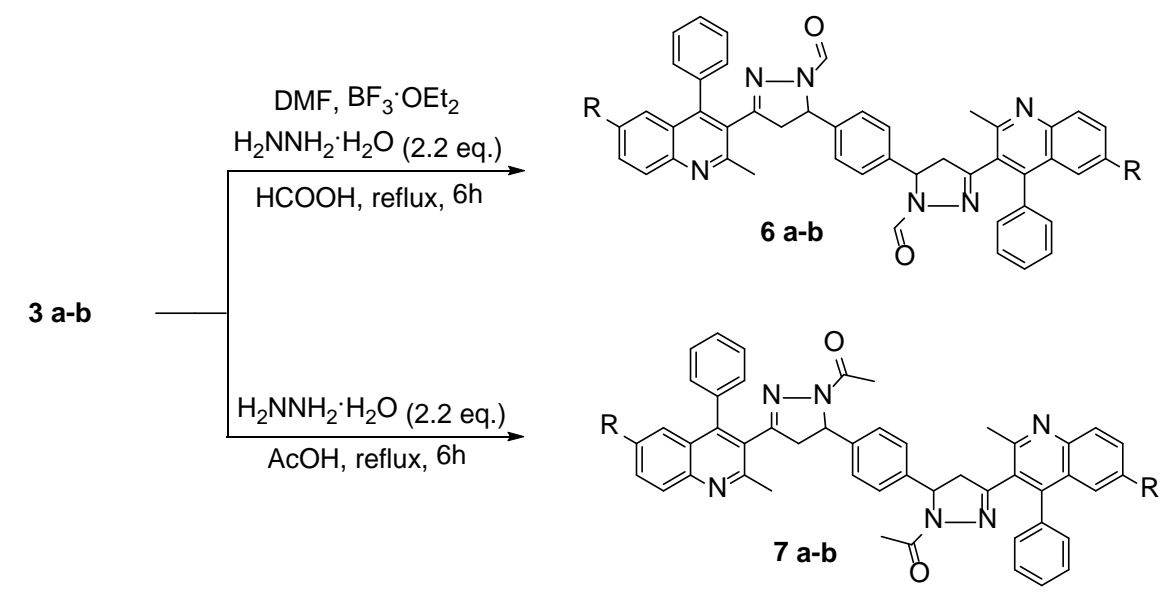

Scheme 3. Synthesis of new bis- $N$-formylpyrazolines 6 and bis- $N$-acetyl pyrazolines 7 .

formic acid or acetic acid. Six hours suffice for complete conversion of the starting materials. It should be noted that $\mathrm{BF}_{3} \cdot \mathrm{Et}_{2} \mathrm{O}$ is required for the conversion of starting materials 2 and 3 to pyrazolines 4 and 6, respectively. All prepared compounds were isolated in good to excellent yields and were characterized by IR, ${ }^{1} \mathrm{HNMR},{ }^{13} \mathrm{CNMR}$ and mass spectral data. The method of preparation of these compounds is very straightforward and free of tedious work up as well is 
quite time saving.

\section{Conclusion}

In conclusion, we have developed an efficient and convenient protocol for the synthesis of novel 2-pyrazoline and bis-2-pyrazoline containing quinoline moiety. The reactions of chalcones and bis-chalcones with hydrazine were carried out at reflux of formic acid in presence of $\mathrm{BF}_{3} \cdot \mathrm{Et}_{2} \mathrm{O}$ or acetic acid without the requirement for an additional catalyst and afforded the desired products from good to excellent yields and in short reaction times. This approach allows a diverse range of compounds to be prepared in good yields, the pharmacological actions of these remaining to be investigated.

\section{Acknowledgements}

We thank Ministère de 1'Enseignement Supérieur et de la Recherche Scientifique for financial support.

\section{References}

[1] De Souza, M.V., Pais, K.C., Kaiser, C.R., Peralta, M.A., Ferreira, M.L. and Lourenço, M.C. (2009) Synthesis and in Vitro Antitubercular Activity of a Series of Quinoline Derivatives. Bioorganic \& Medicinal Chemistry, 17, 1474-1480. https://doi.org/10.1016/j.bmc.2009.01.013

[2] Font, M., Monge, A., Ruiz, I. and Heras, B. (1997) Structure-Activity Relationships in Quinoline Reissert Derivatives with HIV-1 Reverse Transcriptase Inhibitory Activity. Drug Design \& Discovery, 14, 259-272.

[3] Chauhan, P.M. and Srivastava, S.K. (2001) Present Trends and Future Strategy in Chemotherapy of Malaria. Current Medicinal Chemistry, 8, 1535-1542. https://doi.org/10.2174/0929867013371851

[4] Nakamura, T., Oka, M., Aizawa, K., Soda, H., Fukuda, M., Terashi, K., Ikeda, K., Mizuta, Y., Noguchi, Y., Kimura, Y., Tsuruo, T. and Kohno, S. (1999) Direct Interaction between a Quinoline Derivative, MS-209, and Multidrug Resistance Protein (MRP) in Human Gastric Cancer Cells. Biochemical and Biophysical Research Communications, 255, 618-624. https://doi.org/10.1006/bbrc.1999.0245

[5] Kaminsky, D. and Meltzer, R.I. (1968) Quinoline Antibacterial Agents. Oxolinic Acid and Related Compounds. Journal of Medicinal Chemistry, 11, 160-163. https://doi.org/10.1021/jm00307a041

[6] Musiol, R., Jampilek, J., Buchta, V., Silva, L., Niedbala, H., Podeszwa, B., Palka, A., Majerz-Maniecka, K., Oleksyn, B. and Polanski, J. (2006) Antifungal Properties of New Series of Quinoline Derivatives. Bioorganic \& Medicinal Chemistry, 14, 3592-3598. https://doi.org/10.1016/j.bmc.2006.01.016

[7] Warshakoon, N.C., Sheville, J., Bhatt, R.T., Ji, W., Mendez-Andino, J.L., Meyers, K.M., Kim, N., Wos, J.A., Mitchell, C., Paris, J.L., Pinney, B.B., Reizes, O. and Hu, X.E. (2006) Design and Synthesis of Substituted Quinolines as Novel and Selective Melanin Concentrating Hormone Antagonists as Anti-Obesity Agents. Bioorganic \& Medicinal Chemistry Letters, 16, 5207-5211. https://doi.org/10.1016/j.bmcl.2006.07.006

[8] Roma, G., Braccio, M.D., Grossi, G., Mattioli, F. and Ghia, M. (2000) 
1,8-Naphthyridines IV. 9-Substituted $N, N$-Dialkyl-5-(Alkylamino or Cycloalkylamino) $[1,2,4]$ Triazolo[4,3-a] [1,8]Naphthyridine-6-Carboxamides, New Compounds with Anti-Aggressive and Potent Anti-Inflammatory Activities. European Journal of Medicinal Chemistry, 35, 1021-2035. https://doi.org/10.1016/S0223-5234(00)01175-2

[9] Ko, H.H., Tsao, L.T., Yu, K.L., Liu, C.T., Wang, J.P. and Lin, C.N. (2003) Structure-Activity Relationship Studies on Chalcone Derivatives: The Potent Inhibition of Chemical Mediators Release. Bioorganic \& Medicinal Chemistry, 11, 105-112. https://doi.org/10.1016/S0968-0896(02)00312-7

[10] Matsuda, M., Morikawa, T., Ando, S., Iwao. T. and Masayuki, Y. (2003) Structural Requirements of Flavonoids for Nitric Oxide Production Inhibitory Activity and Mechanism of Action. Bioorganic \& Medicinal Chemistry, 11, 1995-2000. https://doi.org/10.1016/S0968-0896(03)00067-1

[11] Herencia, F., Ferrandiz, M.L., Ubeda, A., Dominguez, J.N., Charris, J.E., Lobo. G. M. and Alcaraz, M.J. (1998) Synthesis and Anti-Inflammatory Activity of Chalcone Derivatives. Bioorganic \& Medicinal Chemistry, 8, 1169-1174. https://doi.org/10.1016/S0960-894X(98)00179-6

[12] Ducki, S., Forrest, R., Hadfield, J.A., Kendall, A., Lawrence, N.J., Mcgown, A.T. and Rennison, D. (1998) Potent Antimitotic and Cell Growth Inhibitory Properties of Substituted Chalcones. Bioorganic \& Medicinal Chemistry Letters, 8, 1051-1056. https://doi.org/10.1016/S0960-894X(98)00162-0

[13] Nielsen, S.F., Christensen, S.B., Cruciani, G., Kharazmi. A and Liljefors, T. (1998) Antileishmanial Chalcones: Statistical Design, Synthesis, and Three-Dimensional Quantitative Structure-Activity Relationship Analysis. Journal of Medicinal Chemistry, 41, 4819-4832. https://doi.org/10.1021/jm980410m

[14] Parmer, V.S., Sharma, N.K., Husain, M., Watterson, A.C., Kumar, J., Samuelson, L.A., Ashok, L.C., Prasad, A.K., Kumar, A., Malhotra, S., Kumar, N., Jha, A. Singh, A., Singh, I., Himanshu, V.A., Shakil, N.A., Trikha, S., Mukherjee, S., Sharma, S.K., Singh, S.K., Kumar, A., Jha, H.N., Olsen, C.E., Stove, C.P., Bracke, M.E. and Mareel, M.M. (2003) Synthesis, Characterization and in Vitro Anti-Invasive Activity Screening of Polyphenolic and Heterocyclic Compounds. Bioorganic \& Medicinal Chemistry, 11, 913-929. https://doi.org/10.1016/S0968-0896(02)00539-4

[15] Mukherjee, S., Kumar, V., Prasad, A.K., Raj, H.G., Bracke, M.E., Olsen, C.E., Jain, S.C. and Parmar, V.S. (2001) Synthetic and Biological Activity Evaluation Studies on Novel 1,3-diarylpropenones. Bioorganic \& Medicinal Chemistry, 9, 337-345. https://doi.org/10.1016/S0968-0896(00)00249-2

[16] Lin, Y.M., Zhou, Y., Flavin, M.T., Zhou, L.M., Nie, W. and Chen, F.C. (2002) Chalcones and Flavonoids as anti-Tuberculosis Agents. Bioorganic \& Medicinal Chemistry, 10, 2795-2802. https://doi.org/10.1016/S0968-0896(02)00094-9

[17] Lopez, S.N., Castelli, M.V., Zacchino, S.A., Dominguez, J.N., Lobo, G., Jaime, C.C., Cortes, J.C.G., Ribas, J.C., Devia, C., Ana, M.R. and Ricardo, D.E. (2001) In Vitro Antifungal Evaluation and Structure-Activity Relationships of a New Series of Chalcone Derivatives and Synthetic Analogues, with Inhibitory Properties against Polymers of the Fungal Cell Wall. Bioorganic \& Medicinal Chemistry, 9, 1999-2013. https://doi.org/10.1016/S0968-0896(01)00116-X

[18] Li, R., Kenyon, G.L., Cohen, F.E., Chen, X., Gong, B., Dominguez, J.N., Davidson, E., Kurzban, G., Millar, R.E., Nuzum, E.O., Rosenthal, P.J. and Mckerrow, J.H. (1995) In Vitro Antimalarial Activity of Chalcones and Their Derivatives. Journal of Medicinal Chemistry, 38, 5031-5037. https://doi.org/10.1021/jm00026a010

[19] Liu, M., Wilairat, P. and Go, M.L. (2001) Antimalarial Alkoxylated and Hydrox- 
ylated Chalones: Structure-Activity Relationship Analysis. Journal of Medicinal Chemistry, 44, 4443-4452. https://doi.org/10.1021/jm0101747

[20] Go, M.L., Wu, X. and Liu, X.L. (2005) Chalcones: An Update on Cytotoxic and Chemoprotective Properties. Current Medicinal Chemistry, 12, 483-499. https://doi.org/10.2174/0929867053363153

[21] Johnson, M., Younglove, B., Lee, L., LeBlanc, R., Holt, H., Hills, P., Mackay, H., Brown, T., Mooberry, L.S. and Lee, M. (2007) Design, Synthesis, and Biological Testing of Pyrazoline Derivatives of Combretastatin-A4. Bioorganic \& Medicinal Chemistry, 17, 5897-2901. https://doi.org/10.1016/j.bmcl.2007.07.105

[22] Ramana Reddy, M.V., Billa, V.K., Pallela, V.R., Muralidhar, M.R., Boominathan, R., Gabriel, J.L. and Reddy, E.P. (2008) Design, Synthesis, and Biological Evaluation of 1-(4-Sulfamylphenyl)-3-Trifluoromethyl-5-Indolylpyrazolines as Cyclooxygenase-2 (COX-2) and Lipoxygenase (LOX) Inhibitors. Bioorganic \& Medicinal Chemistry, 16, 3907-3916. https://doi.org/10.1016/j.bmc.2008.01.047

[23] Bhat, A.R., Athar, F. and Azam, A. (2009) New Derivatives of 3,5-Substituted-1,4,2-Dioxazoles: Synthesis and Activity against Entamoeba histolytica. European Journal of Medicinal Chemistry, 44, 926-936. https://doi.org/10.1016/j.ejmech.2008.02.001

[24] Ozdemir, Z., Kandilci, H.B., Gumusel, B.H., Calıs, U. and Bilgin, A.A. (2006) Synthesis and Studies on Antide Pressant and Anticonvulsant Activities of Some 3-(2-Furyl)-Pyrazoline Derivatives. European Journal of Medicinal Chemistry, 42, 373-379. https://doi.org/10.1016/j.ejmech.2006.09.006

[25] Ozdemir, A., Zitouni, G.T., Kaplancikl, Z.A., Revial, G. and Guven, K. (2007) Synthesis and Antimicrobial Activity of 1-(4-Aryl-2-Thiazolyl)-3-(2-Thienyl)-5-Aryl-2-Pyrazoline Derivatives. European Journal of Medicinal Chemistry, 42, 403-409. https://doi.org/10.1016/j.ejmech.2006.10.001

[26] Agarwal, Y.K., Manna, K., Bhatt, H., Gogai, P., Babu, V.H. and Srinivasan, K.K. (2007) Synthesis and Biological Evaluations of New Benzofuran 1,3,5-Trisubstituted Pyrazoline Derivatives of Paracetamol as Potential Antitubercular, Antimicrobial Agents. Indian Journal of Heterocyclic Chemistry, 16, 263-266.

[27] Carrion, M.D., Luisa, C., Lopez, L.C., Camacho, M.E., Tapias, V., Escames, G., Castroviejo, D.A., Espinosa, A., Gallo, M.A. and Entrena, A. (2008) Pyrazoles and Pyrazolines as Neural and Inducible Nitric-Oxide Synthase (nNOS and iNOS) Potential Inhibitors (III). European Journal of Medicinal Chemistry, 43, 2579-2591. https://doi.org/10.1016/j.ejmech.2008.01.014

[28] Wang, G.W., Jia, C.S. and Dong, Y.W. (2006) Benign and Highly Efficient Synthesis of Quinolines from 2-Aminoarylketone or 2-Aminoarylaldehyde and Carbonyl Compounds Mediated by Hydrochloric Acid in Water. Tetrahedron Letters, 47, 1059-1063. https://doi.org/10.1016/j.tetlet.2005.12.053

[29] Fun, H.K., Loh, W.S., Sarveswari, S., Vijayakumar, V. and Reddy, B.P. (2009) 1-(6-Chloro-2-Methyl-4-Phenyl-3-Quinolyl)Ethanone. Acta Crystallographica, E65, o2688-o2689. https://doi.org/10.1107/S1600536809040306

[30] Kotra, V., Ganapaty, S. and Adapa, S.R. (2010) Synthesis of a New Series of Quinolinyl Chalcones as Anticancer and Anti-Inflammatory Agents. Indian Journal of Chemistry (Section B), 49, 1109-1116.

[31] Kedjadja, A., Merdes, R., Bouacida, S., Roisnel, T. and Belfaitah, A. (2013) (2E,2'E)-1,1'-Bis(6-Chloro-2-Methyl-4-Phenylquinolin-3-yl)-3,3'-(1,4-Phenylene)Di prop-2-en-1-One Ethyl Acetate Disolvate. Acta Crystallographica Section E, 69, o37-o38. https://doi.org/10.1107/S1600536812049422 